\title{
Tendência temporal da inserção de nutricionistas no Sistema Único de Saúde segundo regiões do Brasil no período de 2009 a 2018
}

\author{
Letícia Batista de Azevedo* \\ Erika Cardoso dos Reis**
}

\begin{abstract}
Resumo
O campo científico da nutrição emerge com maior intensidade no início do século XX, sendo considerado um fenômeno relativamente recente. No Brasil, a formação do nutricionista teve início na década de 1940, quando se obteve a primeira geração de médicos nutrólogos e a criação dos quatro primeiros cursos do país. Estudos sobre a inserção dos nutricionistas no âmbito do SUS são escassos. Assim, o presente artigo tem por objetivo avaliar a tendência temporal da inserção de nutricionistas nos Sistema Único de Saúde no período de 2009 a 2018. Trata-se de um estudo observacional, descritivo, realizado a partir de dados secundários fornecidos pelo IBGE e CNES. A análise dos dados possibilitou a obtenção da quantidade e distribuição de nutricionistas por região/unidade da federação e ano de competência. A tabulação dos dados foi realizada no TabWin versão 4.15 e as análises foram realizadas com o uso dos Softwares Microsoft Excel 2016® e Stata 15.0. Observou-se uma tendência temporal de aumento do número de nutricionistas no país com velocidade maior de crescimento na região nordeste e menor na região sudeste, que registrou a maior densidade de profissionais. A região norte apresentou o menor número de nutricionistas. Acredita-se que o perfil observado pode estar relacionado com a oferta salarial, com características específicas e com a intensidade de criação dos cursos de nutrição em cada região.
\end{abstract}

Palavras-chaves: Nutricionistas. Política Nacional de Alimentação e Nutrição. Vigilância Nutricional. Atenção à Saúde.

\section{INTRODUÇÃO}

O percurso histórico do campo de atuação nutricional é relativamente recente com manifestações oriundas do início do século $X X$. No Brasil, a formação de nutricionista começou na década de 40, quando se obteve a primeira geração de médicos nutrólogos e a criação dos quatro primeiros cursos do país, sendo um em São Paulo e três no Rio de Janeiro' ${ }^{1}$.

Com a fundação da Sociedade Brasileira de Nutrição (1940), a criação dos Arquivos Brasileiros de Nutrição (1944) e a fundação da Associação Brasileira de Nutricionistas
(ABN) (1949) pode-se dizer que a Nutrição inicia seu processo de institucionalização no Brasil enquanto ciência, área de política pública e campo profissional' ${ }^{1}$.

Apesar da institucionalização da profissão ter sido na década de 40, o reconhecimento do curso de nutrição como formação em nível superior veio a ocorrer com o Parecer no 265, de 19 de outubro de 1962, onde o então Conselho Federal de Educação (CFE) reconheceu os Cursos de Nutricionistas como de nível superior ${ }^{1,2}$.

Em paralelo a essa institucionalização 
do campo da nutrição no Brasil, a luta pela saúde apresenta um crescimento significativo, liderada pelo movimento pela reforma sanitária ${ }^{3}$. Assim, após o reconhecimento do direito à saúde pela Constituição Cidadã de 1988, ocorre a criação do Sistema Único de Saúde (SUS), a fim de garantir assistência igualitária à saúde dos indivíduos mediante a implementação de ações na área da saúde, tornando, portanto, obrigatório o atendimento gratuito a toda população $0^{4,5}$.

A partir da Lei $8.080^{5}$ torna-se então dever do Estado garantir a saúde mediante a execução de políticas econômicas e sociais que visem a redução de riscos de doenças e de outros agravos e do estabelecimento de condições que assegurem acesso universal e igualitário às ações e aos serviços para a promoção, proteção e recuperação da saúde. Para ofertar as ações de promoção, proteção e recuperação da saúde, o sistema de saúde encontra-se organizado em níveis de complexidade distintos a fim de atender as diferentes demandas da população ${ }^{6,7}$.

A presença do nutricionista nos serviços de saúde contribuem de maneira importante para atuar diretamente junto a indivíduos, famílias e comunidade; na participação de ações de educação continuada de profissionais de saúde; na articulação de estratégias de ação com os equipamentos sociais de seu território de atuação, em prol da promoção da alimentação saudável, do Direito Humano à Alimentação Adequada e da Segurança Alimentar e Nutricional ${ }^{8}$. Além disso, este profissional tem como funções específicas: a avaliação e monitoramento dos determinantes de nutrição e saúde, desenvolvimento de estratégias de educação continuada em alimentação e nutrição direcionadas aos profissionais de saúde, avaliação dos resultados e impacto dos programas e ações de nutrição e saúde, entre outras ${ }^{9}$.

Diversos autores vem reforçando o quanto a presença do nutricionista pode apoiar as ações de atenção à saúde da população para o fortalecimento das atividades de promoção de práticas alimentares saudáveis e de assistência nutricional ${ }^{10-12}$. Assim, o nutricionista, como profissional de saúde, está inserido em todos os níveis de complexidade do serviço de saúde - da atenção primária à atenção hospitalar - e sua atuação tem contribuído para promoção, proteção, tratamento e manutenção da saúde.

Essa inserção do profissional nutricionista no sistema de saúde contribui no processo coletivo de trabalho em prol da promoção da saúde e se faz necessária para o enfrentamento do complexo perfil nutricional apresentado pela população brasileira, onde a desnutrição expressa por déficit de crescimento e carências nutricionais por micronutrientes coexiste com aumento da obesidade e de outras doenças crônicas não transmissíveis associadas à alimentação $(\mathrm{DCNT})^{13}$. Deste modo, a presença do nutricionista pode contribuir para a qualificação das ações de atenção nutricional auxiliando as pessoas em todas as fases do curso da vida a desenvolverem práticas alimentares adequadas, mediante a assistência a indivíduos e grupos populacionais. Esta contribuição do profissional nutricionista está prevista na Resolução do Conselho Federal de Nutricionistas (CFN) 600/2018 ${ }^{14}$ e na Política Nacional de Alimentação e Nutrição (PNAN) $)^{15}$, marcos institucionais que reiteram a importância deste profissional na ampliação dos conhecimentos sobre a alimentação e nutrição e na promoção de práticas alimentares saudáveis ${ }^{7,8}$.

Portanto, considerando que o nutricionista representa uma categoria profissional com contribuições técnicas e científicas específicas e significativas para a organização da atenção nutricional, desde a atenção primária até a atenção especializada hospitalar, que incluem a promoção da saúde e da alimentação adequada e saudável, a vigilância alimentar e nutricional e ações de prevenção e tratamento de doenças em todas as fases da vida, o objetivo deste artigo é o de avaliar a tendência temporal da inserção de nutricionistas no Sistema Único de Saúde (SUS) no período de 2009 a 2018. 


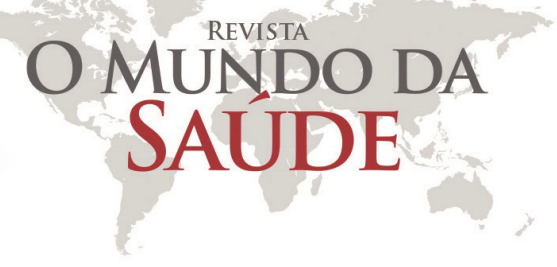

\section{MÉTODOS}

Trata-se de um estudo observacional, descritivo, baseado em dados secundários, de acesso livre. Os dados referentes à quantidade total de profissionais nutricionistas registradas nos Conselhos Regionais de Nutricionistas foram extraídos da página eletrônica do $\mathrm{CFN}^{16}$, e a quantidade de profissionais nutricionistas vinculados a um serviço de saúde - público ou privado - foram obtidos no Cadastro Nacional de Estabelecimentos de Saúde (CNES). A quantidade total de nutricionistas no CNES foi obtida por meio da seleção "Região/ Unidade da Federação" e "Ano/mês competência " e "Ocupações de Nível Superior: Nutricionista" e para a obtenção dos dados da quantidade de nutricionistas inseridas no SUS foi acrescentado a seleção "Atende no SUS: Sim"17. O tamanho da população anual foi obtido na página eletrônica do Instituto Brasileiro de Geografia e Estatística $(\text { IBGE })^{18}$.

As etapas deste estudo consistiram em uma busca inicial da quantidade de nutricionistas no Brasil por região na base de dados do CFN, que é divulgada por meio de relatórios trimestrais. A quantidade de nutricionistas para cada ano de análise registradas no CFN foi obtida por meio da média dos quatro trimestres de cada ano. Do mesmo modo, sendo a base de dados do CNES mensal, foram extraídos os dados dos 12 meses de cada ano e a média anual foi utilizada para análise da quantidade de nutricionistas vinculados à um serviço de saúde no referido ano. Primeiro foi extraído a quantidade de nutricionistas em todos

\section{RESULTADOS}

No ano de 2009, de acordo com os dados de inscrição ativas no CFN, havia uma média de 14.502 nutricionistas no Brasil e em 2018 esse número era de 30.226, o que representa um crescimento de 2,1 vezes. A tabela 1 apresenta os dados relativos à quantidade de nutricionistas por região do Brasil no período 2009 a 2018. os estabelecimentos de saúde e posteriormente selecionado apenas os estabelecimentos que atendem no SUS. E por fim, foram obtidos os dados da população na página eletrônica do IBGE - censo de 2010 e estimativa anual dos demais anos - e calculada a distribuição dos profissionais nutricionistas.

Foi analisado a quantidade de nutricionistas total, a quantidade de nutricionistas no serviço público e privado para cada 100 mil habitantes a cada ano, e a tendência temporal da distribuição destes profissionais nos serviços que atendem no SUS segundo Região do Brasil. A análise da taxa de nutricionistas por 100 mil habitantes foi realizada para permitir comparabilidade entre as regiões do país.

Foi realizada a análise de tendência temporal, utilizaram-se os procedimentos de regressão linear generalizada de Prais-Winsten, que considera a autocorrelação serial. Para isso, estimou-se a variação percentual anual (em inglês, anual percent change [APC]) e seus respectivos intervalos de confiança de 95\% (IC95\%). Os valores de $p$ foram obtidos pelo teste t com nível de significância estatística de 5\%. Para tabulação, análise descritiva e cálculo das taxas, foi utilizado o Software Microsoft Office Excel 2016, e para análise de tendência, o Stata 15.0.

Por utilizar apenas dados disponíveis publicamente e sem identificação dos sujeitos, foi dispensada a submissão ao Comitê de Ética em Pesquisa (CEP).

Em estabelecimentos que atendem no SUS, esse quantitativo foi de 9.864 em 2009 passando para 21.385 em 2018.

Em 2018 a maior concentração de profissionais estava na região sudeste (16.269), seguida das regiões sul (7.224), nordeste (3.217), centro-oeste (2.638) e norte (877). A região 
norte apresentou um quantitativo 18,5 vezes menor que o da região sudeste. As regiões nordeste e centro-oeste foram as regiões com maior aumento de nutricionistas, registrados em 3,5 e 2,3, respectivamente.

Na tabela 2 está apresentado a quantidade total de nutricionistas por 100 mil habitantes no período de 2009 a 2018 registradas no CFN, segundo regiões do Brasil. A quantidade de profissionais nutricionistas registrados no período avaliado variou de 8 para 14. Notase que as regiões sul e sudeste apresentaram maior quantidade de nutricionistas por 100 mil habitantes, enquanto na região norte verificou-se a menor quantidade de profissionais registrados no CFN.

As regiões nordeste e centro-oeste foram as que apresentaram maior crescimento de profissionais no período analisado, 3,27 e 2,00 respectivamente (tabela 2 ).

A tabela 3 se refere ao número de nutricionistas cadastrados no CNES por 100 mil habitantes. Os profissionais nutricionistas cadastrados no CNES são aqueles que exercem função em estabelecimentos públicos ou privados na área da saúde. A região sul conta com mais profissionais cadastrados no CNES por 100 mil habitantes $(16,07)$, e a norte com a menor $(8,65)$. Ao longo do período analisado, o número de nutricionistas cadastrados no CNES variou de 6,31 para 13,85 por 100 mil habitantes no Brasil.

Quanto ao aumento nas regiões, observou-se que a região nordeste obteve o maior aumento de nutricionistas cadastrados (2,54 vezes), e a região sudeste a que apresentou menor incremento (2,04 vezes).

Em relação aos nutricionistas cadastrados em serviços que atendem no SUS no CNES por 100 mil habitantes, o gráfico 1 apresenta a evolução ao logo do período analisado e a tabela 4 mostra que houve uma variação de 5,15 para 10,26 profissionais entre as regiões do Brasil. Em 2009, as regiões norte e nordeste possuíam a menor quantidade de nutricionistas em estabelecimentos que atendem no SUS por 100 mil habitantes. Em 2018, a região nordeste concentrava o maior número de profissionais nutricionistas no SUS por 100 mil habitantes $(11,83)$ sendo esta região a que mais teve aumento $(2,4)$; em seguida a região sul $(11,15)$ com aumento de 2,0 ; e a região norte com menor quantidade de profissionais $(7,53)$ com aumento de 2,1. A análise da tendência temporal mostra que houve crescimento da inserção do nutricionista no SUS em todas as regiões do Brasil com maior variação percentual anual na região nordeste $(8,90)$, seguida da região centro-oeste $(7,62)$, e menor na região sudeste $(5,35)$.

Tabela 1 - Quantidade média de nutricionistas registrados no Conselho Federal de Nutricionistas por região do país no período de 2009 a 2018.

\begin{tabular}{lccccccccccc}
\hline Região & 2009 & 2010 & 2011 & $\mathbf{2 0 1 2}$ & $\mathbf{2 0 1 3}$ & $\mathbf{2 0 1 4}$ & $\mathbf{2 0 1 5}$ & $\mathbf{2 0 1 6}$ & $\mathbf{2 0 1 7}$ & $\mathbf{2 0 1 8}$ & Aumento \\
\hline Região Norte & 501 & 586 & 714 & 849 & 992 & 1106 & 637 & 740 & 824 & 877 & 1,8 \\
Região Nordeste & 929 & 1089 & 1261 & 1446 & 1667 & 1914 & 2229 & 2538 & 2923 & 3217 & 3,5 \\
Região Sudeste & 8224 & 9282 & 10245 & 11208 & 11874 & 12722 & 13587 & 14380 & 15142 & 16269 & 2,0 \\
Região Sul & 3710 & 4139 & 4601 & 4930 & 5408 & 5772 & 5997 & 6424 & 6808 & 7224 & 1,9 \\
Região Centro-Oeste & 1139 & 1316 & 1491 & 1686 & 1834 & 2013 & 2183 & 2326 & 2459 & 2638 & 2,3 \\
Total/Brasil & 14502 & 16411 & 18313 & 20118 & 21775 & 23527 & 24633 & 26407 & 28156 & 30226 & 2,1 \\
\hline
\end{tabular}

Fonte: Conselho Federal de Nutricionistas, 2020. 


\section{O MUNDDO DA \\ SAUDE}

Tabela 2 - Taxa de nutricionistas registrados no Conselho Federal de Nutricionistas por 100 mil habitantes, segundo região do país no período de 2009 a 2018.

\begin{tabular}{lccccccccccc}
\hline Região & $\mathbf{2 0 0 9}$ & $\mathbf{2 0 1 0}$ & $\mathbf{2 0 1 1}$ & $\mathbf{2 0 1 2}$ & $\mathbf{2 0 1 3}$ & $\mathbf{2 0 1 4}$ & $\mathbf{2 0 1 5}$ & $\mathbf{2 0 1 6}$ & $\mathbf{2 0 1 7}$ & $\mathbf{2 0 1 8}$ & Aumento \\
\hline Região Norte & 3,26 & 3,60 & 4,44 & 5,21 & 5,84 & 6,42 & 3,64 & 4,18 & 4,59 & 4,82 & 1,48 \\
Região Nordeste & 1,73 & 2,01 & 2,36 & 2,68 & 2,99 & 3,41 & 3,94 & 4,46 & 5,10 & 5,67 & 3,27 \\
Região Sudeste & 10,16 & 11,30 & 12,65 & 13,74 & 14,06 & 14,95 & 15,85 & 16,65 & 17,42 & 18,55 & 1,82 \\
Região Sul & 13,38 & 14,82 & 16,69 & 17,79 & 18,78 & 19,89 & 20,52 & 21,82 & 22,96 & 24,28 & 1,81 \\
Região Centro-Oeste & 8,20 & 9,17 & 10,47 & 11,69 & 12,23 & 13,23 & 14,14 & 14,85 & 15,49 & 16,40 & 2,00 \\
Total/Brasil & 7,57 & 8,42 & 9,52 & 10,38 & 10,83 & 11,60 & 12,05 & 12,81 & 13,56 & 14,50 & 1,91 \\
\hline
\end{tabular}

Fonte: Conselho Federal de Nutricionistas, 2020.

Tabela 3 - Taxa de nutricionistas cadastrados no CNES por 100 mil habitantes, segundo região do país no período de 2009 a 2018.

\begin{tabular}{lccccccccccc}
\hline Região & $\mathbf{2 0 0 9}$ & $\mathbf{2 0 1 0}$ & $\mathbf{2 0 1 1}$ & $\mathbf{2 0 1 2}$ & $\mathbf{2 0 1 3}$ & $\mathbf{2 0 1 4}$ & $\mathbf{2 0 1 5}$ & $\mathbf{2 0 1 6}$ & $\mathbf{2 0 1 7}$ & $\mathbf{2 0 1 8}$ & Aumento \\
\hline Região Norte & 4,06 & 4,40 & 4,97 & 5,41 & 5,81 & 6,54 & 6,89 & 7,41 & 8,01 & 8,65 & 2,13 \\
Região Nordeste & 5,36 & 6,14 & 6,95 & 7,74 & 8,43 & 9,65 & 10,35 & 11,18 & 12,15 & 13,60 & 2,54 \\
Região Sudeste & 6,92 & 7,78 & 8,78 & 9,56 & 10,12 & 11,24 & 11,84 & 12,52 & 13,22 & 14,12 & 2,04 \\
Região Sul & 7,40 & 8,36 & 9,54 & 10,69 & 11,22 & 12,40 & 13,26 & 14,14 & 15,09 & 16,07 & 2,17 \\
Região Centro-Oeste & 6,76 & 7,86 & 9,12 & 10,06 & 10,73 & 11,79 & 12,73 & 13,68 & 13,93 & 15,00 & 2,22 \\
Total/Brasil & 6,31 & 7,13 & 8,09 & 8,90 & 9,49 & 10,61 & 11,28 & 12,03 & 12,79 & 13,85 & 2,19 \\
\hline
\end{tabular}

Fonte: Cadastro Nacional de Estabelecimentos de Saúde, 2020. 


\begin{tabular}{|c|c|c|c|c|c|c|c|c|c|c|c|c|c|c|}
\hline Região & 2009 & 2010 & 2011 & 2012 & 2013 & 2014 & 2015 & 2016 & 2017 & 2018 & APC & IC $95 \%$ & $\mathrm{p}$-valor & Tendência \\
\hline Norte & 3,65 & 3,92 & 4,44 & 4,82 & 5,10 & 5,79 & 6,05 & 6,45 & 6,93 & 7,53 & 6,93 & $\begin{array}{l}6,46- \\
7,40\end{array}$ & $<0,001$ & Crescente \\
\hline Nordeste & 4,88 & 5,57 & 6,28 & 6,97 & 7,56 & 8,68 & 9,29 & 9,88 & 10,64 & 11,83 & 8,90 & $\begin{array}{l}8,02- \\
9,78\end{array}$ & $<0,001$ & Crescente \\
\hline Sudeste & 5,44 & 6,05 & 6,72 & 7,20 & 7,54 & 8,25 & 8,49 & 8,71 & 8,89 & 9,34 & 5,35 & $\begin{array}{l}3,78- \\
6,93\end{array}$ & $<0,001$ & Crescente \\
\hline Sul & 5,61 & 6,21 & 6,95 & 7,79 & 8,07 & 8,97 & 9,53 & 9,99 & 10,54 & 11,15 & 6,96 & $\begin{array}{l}5,78- \\
8,16\end{array}$ & $<0,001$ & Crescente \\
\hline $\begin{array}{l}\text { Centro- } \\
\text { Oeste }\end{array}$ & 5,26 & 6,00 & 6,82 & 7,51 & 8,02 & 8,88 & 9,65 & 10,32 & 10,71 & 11,11 & 7,62 & $\begin{array}{l}5,92- \\
9,34\end{array}$ & $<0,001$ & Crescente \\
\hline Brasil & 5,15 & 5,76 & 6,45 & 7,04 & 7,45 & 8,31 & 8,74 & 9,14 & 9,58 & 10,26 & 6,88 & $\begin{array}{l}5,68- \\
8,09\end{array}$ & $<0,001$ & Crescente \\
\hline
\end{tabular}

Fonte: Cadastro Nacional de Estabelecimentos de Saúde, 2020.

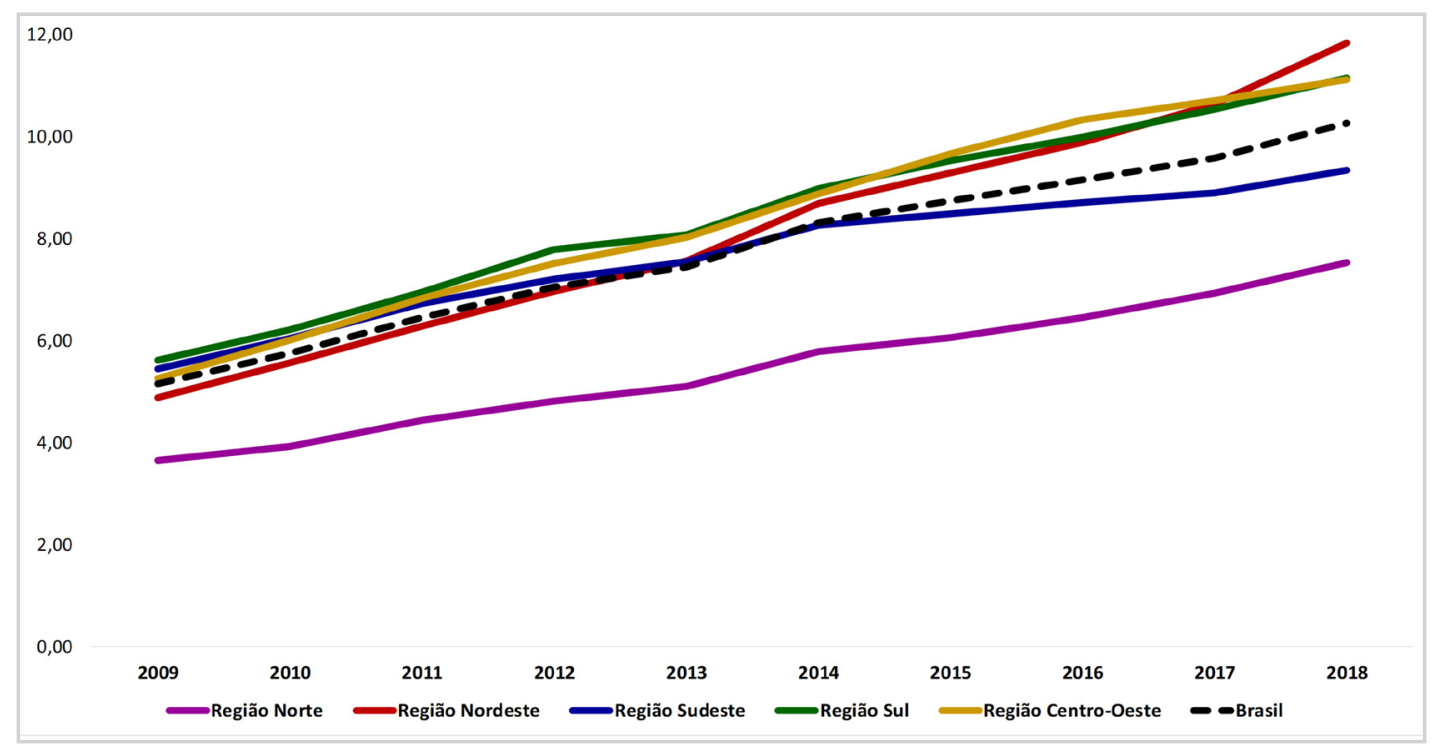

Fonte: Elaborado pelas autoras, a partir de dados do Cadastro Nacional de Estabelecimentos de Saúde e IBGE, 2020.

Figura 1 - Evolução da taxa de nutricionistas cadastrados no CNES em serviços que atendem o SUS por 100.000 habitantes, segundo região do país no período de 2009 a 2018.

\section{DISCUSSÃO}

O campo da nutrição pode ser considerado novo e por isso enfrenta muitos desafios. Tais desafios podem ser percebidos no complexo processo de formação dos profissionais nutricionistas, que envolvem aspectos conceituais, e também relações institucionais de enfrentamento de conhecimentos e de valores cristalizados e hegemônicos ${ }^{19}$.
Os dados mostram que em 2009 existiam apenas 14.502 nutricionistas no país, quantidade esta que em 2018 passou a ser de 30.226 profissionais. Apesar da notória diferença entre as regiões, sendo a região sudeste a que possui o maior número de nutricionistas no Brasil, as regiões com crescimento maior registrado foram as regiões nordeste (3,5 vezes) e centro-oeste 
(2,3 vezes) e menor se encontrou na região norte ( 1,8 vezes). O estudo realizado por Haddad et al, também identifica que na análise dos cursos de graduação em nutrição, no período ente 2006 e 2008, o Nordeste foi a região que apresentou maior quantidade de concluintes por habitantes quando comparados às demais regiões ${ }^{20}$.

Estes dados corroboram com os resultados de Neves et al. ${ }^{21}$, e também com os achados da Estação de Trabalho da Rede Observatório de Recursos Humanos em Saúde do Instituto de Medicina Social da Universidade do Estado do Rio de Janeiro ${ }^{22}$, que em sua pesquisa realizada no período de 2000 a 2010 observou maior percentual de variação no número de cursos de nível superior em nutrição nas regiões Nordeste $(757 \%)$, enquanto a região Centro-Oeste obteve incremento de $267 \%$, a Sudeste de $223 \%$ e a Sul de $146 \%$. Apesar da região norte apresentar os menores índices de nutricionistas em comparação as outras regiões, o percentual de variação é de $700 \%$. Este fato pode estar relacionado ao número de cursos de nutrição abertos nas regiões desde a implantação do curso de nutrição no Brasil até os dias de hoje.

$\mathrm{Na}$ região norte, entre o primeiro e segundo curso de nutrição aberto há uma diferença de 26 anos (o primeiro criado em 1973 e o segundo em 1999) ${ }^{23}$. Na região Centro-Oeste, o primeiro curso foi criado em 1975, em 1996 havia 4 cursos e em 200930 cursos. Na região Nordeste, entre 1976 e 1986, ocorreu a criação de mais seis cursos nos estados da Paraíba (1976), Piauí (1976), Rio Grande do Norte (1976), Ceará (1977), Alagoas (1978) e Bahia (1986), de modo que até 1996 existiam 8 cursos, e em 2009 são encontrados 58 cursos em todos os estados da região ${ }^{23}$. Já na região Sudeste, até 1996 existiam 23 cursos, e em 2009, $221^{23}$.

Outra possível explicação para à crescente do número de cursos de nutrição na região nordeste é que a mesma apresenta o maior salário. Em Sergipe, o piso salarial do nutricionista é de $\mathrm{R} \$$ 5.162,85 (referente à 5,4 salários mínimos), sendo este o maior salário de nutricionista no Brasil. Enquanto o estado do Espírito Santo (região sudeste) apresenta o menor piso salarial, com R\$2.400,00 (2,5 salários mínimos) ${ }^{24}$.
A explicação para a tendência crescente do número de cursos de nutrição no Brasil pode estar relacionada também a uma liberalização para abertura de cursos de graduação, uma vez que haviam apenas 45 cursos de nutrição até o ano 1996, e após a promulgação da Lei de Diretrizes e Bases da Educação Nacional (LDB) ${ }^{25}$, que impulsionou o ensino privado, o aumento de cursos torna-se evidente. No Brasil, entre 1996 e janeiro de 2000, haviam 106 cursos de nutrição, em agosto de 2009 o número de cursos era de $391^{23}$, em 2018 havia 609 cursos $^{26}$ e em 2020 a quantidade de cursos ultrapassa $800^{27}$.

Pinheiro et al. ${ }^{28}$ realizaram um estudo que mostra que a saúde pública é reconhecida pelos estudantes como uma das principais áreas de interesse na educação de nutricionistas. Porém, em 2008, um estudo com a participação de 587 profissionais das cinco regiões do Brasil inferiu que somente $10,2 \%$ desses trabalham nessa área ${ }^{29}$. Ao estudar o perfil profissional dos nutricionistas egressos de uma universidade paulista, Gambardella et al..$^{30}$ observaram que 36,6\% atuavam em Nutrição Clínica, 31,0\% em unidades de alimentação e nutrição e 7,0\% em Saúde Pública. Em estudo conduzido com recém egressos também de uma universidade paulista, Miranda et al. ${ }^{31}$ encontraram que $56,6 \%$ atuavam na área de Nutrição Clínica e 53,8\% na área de Alimentação Coletiva; desses, 7,2\% atuavam na área de Saúde Pública. Dados mais recentes do CFN (2019) mostram que 17,7\% dos nutricionistas atuam na área de saúde coletiva ${ }^{26}$.

Como observado, o número de nutricionistas registrados em serviços que atendem o SUS no CNES em 2018 era de 21.385 (16,15\%), e considerando que entre os estabelecimentos que atendem no SUS podem estar aqueles em que os nutricionistas trabalham especificamente com a nutrição clínica, conclui-se que é muito provável que a quantidade de profissionais que atuam exclusivamente na saúde coletiva pode ser ainda menor. É necessário ainda destacar a importância do profissional nutricionista na gestão das políticas e programas de saúde pública conforme definido pela Resolução CFN 600/201814, uma importante área da saúde coletiva. Embora esta resolução não determine 


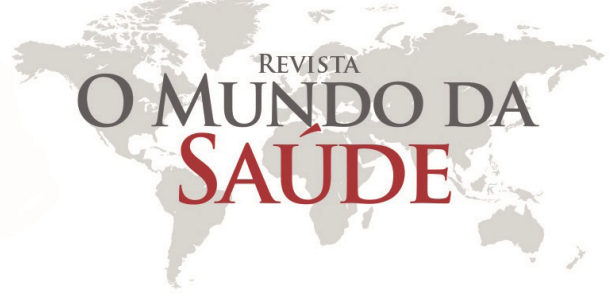

a quantidade mínima de nutricionistas para o atendimento das demandas de saúde pública, ela orienta a necessidade de 01 (um) nutricionista por programa ${ }^{14}$, o que é difícil de mensurar por meio dos dados apresentados CNES por não ser possível conhecer quais e quantos programas vigentes existem na esfera municipal.

A análise baseada no tamanho da população identifica que em 2009 havia 7,57 nutricionistas/100.000 habitantes e em 2018 14,50 nutricionistas/100.000 habitantes. Considerando a importância deste profissional discutida por outros diferentes autores, pode-se considerar que ainda esse número encontra-se abaixo da quantidade de profissionais necessária na rede de atenção à saúde, em especial na atenção primária ${ }^{10,32}$, importante local de atuação do nutricionista na saúde coletiva.

Com a criação das equipes do Núcleo de Apoio a Saúde da Família (eNASF) ${ }^{33}$ houve aumento no quantitativo de nutricionistas que atuam na rede básica de saúde, porém essa quantidade reduzida pode ser entendida pelo fato da inserção desse profissional depender da decisão do gestor municipal em termos da abertura de concurso público ou processo seletivo para suprir essa demanda. Para os gestores decidirem sobre sua inclusão, além de considerar a necessidade do profissional, também seria preciso avaliar a viabilidade e ordenar as prioridades de gastos estabelecidos para a área de saúde do município.

No entanto, apesar do número ainda insuficientes de profissionais, a presença do nutricionista na atenção primária é justificada pela necessidade de ações de atenção nutricional de forma qualificada, como descrita na Política Nacional de Alimentação e Nutrição
$(\mathrm{PNAN})^{34}$. Promover ações de nutrição com a abordagem da saúde coletiva consiste em uma forma estratégica de promoção da saúde e de prevenção de doenças associadas a uma alimentação inadequada, tendo em vista que, as complicações advindas desses casos resultam em uma maior demanda nos serviços de saúde de média e alta complexidade e também acarretam em elevados gastos públicos para serem tratados. Com isso, torna-se imprescindível a presença de um profissional capacitado na área de nutrição para abordar e socializar o conhecimento sobre o acesso e o consumo de alimentos mais saudáveis e de forma intersetorial e com o apoio de políticas públicas adequadas promover a realização de ações que promovam a segurança alimentar e nutricional à população ${ }^{35}$.

Este estudo traz uma análise fundamental sobre a tendência e evolução da quantidade de nutricionistas na área da saúde e sua distribuição nas regiões do país, o que contribui para a gestão em saúde refletir sobre a implementação de estratégias de incentivo e apoio nas áreas que mais necessitam deste profissional. As suas limitações se referem ao uso das bases de dados secundárias, a partir de dados informados pelos gestores responsáveis pelos estabelecimentos de saúde que, a depender de fatores alheios a presença do profissional, pode ser enviado à base federal com atrasos. Além disso, a análise da taxa de nutricionistas nos estabelecimentos que atendem o SUS não permitem um detalhamento da sua inserção (na gestão ou assistência), uma vez que muitas nutricionistas destinam parte da sua carga horária para a gestão de programas e assistência à saúde, principalmente em municípios de porte populacional menor.

\section{CONCLUSÃO}

Diante do exposto, conclui-se que a quantidade de nutricionistas no país inseridos na área da saúde, em especial no SUS, vem crescendo de forma acelerada. Porém é importante ressaltar que essa quantidade ainda é bem desigual e insuficiente para as demandas existentes quando se analisa a distribuição do profissional proporcionalmente ao tamanho da população e à prevalência dos problemas de saúde relacionados à questão alimentar e nutricional. É preciso reconhecer e divulgar a contribuição do nutricionista na promoção da saúde da população e no incentivo aos modos de vida saudável para que os gestores 


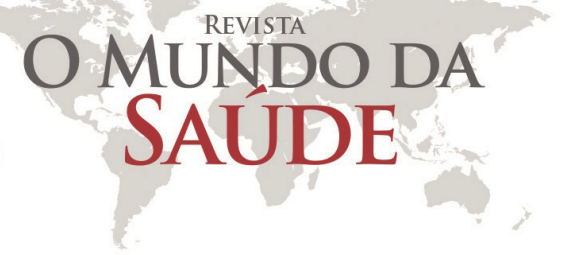

públicos realizem maiores esforços em viabilizar a integração desse profissional às equipes de saúde e na gestão municipal, estadual e federal da área de alimentação e nutrição.

\section{REFERÊNCIAS}

1. Associação Brasileira de Nutrição. Histórico do nutricionista no Brasil, 1939 a 1989: coletânea de depoimentos e documentos. São Paulo, S.P.: Atheneu Editora São Paulo; 1991.

2. Vasconcelos F de AG de. O nutricionista no Brasil: uma análise histórica. Rev Nutr. agosto de 2002;15(2):127-38. http://dx.doi. org/10.1590/S1415-52732002000200001.

3. Arouca AS da S. Democracia é saúde. In: CONFERÊNCIA NACIONAL DE SAÚDE, 8. Anais da 8a Conferência Nacional de Saúde. Brasília: Centro de Documentação do Ministério da Saúde. 1986;35-42.

4. Conselho Nacional de Secretarias Municipais de Saúde (Brazil), organizador. O SUS de A a Z: garantindo saúde nos municípios. 3a. ed. Brasília, DF: Ministério da Saúde, Conselho Nacional de Secretarias Municipais de Saúde; 2009. 447 p. (Série F-Comunicação e educação em saúde).

5. Brasil. Lei no 8.080, de 19 de setembro de 1990. Dispõe sobre as condições para a promoção, proteção e recuperação da saúde, a organização e o funcionamento dos serviços correspondentes e dá outras providências. [Internet]. 1990. Disponível em: http://www. planalto.gov.br/ccivil_03/leis/l8080.htm

6. Mendes EV. A construção social da atenção primária à saúde. Brasília: Conselho Nacional de Secretários de Saúde - CONASS. $2015 ; 194$.

7. Mendes EV. As redes de atenção à saúde. Brasília: Organização Pan-Americana da Saúde,; 2011. 549 p.: il.

8. Recine $E$, Leão $M$, Carvalho $M$ de $F$. O papel do nutricionista na atenção primária à saúde [organização Conselho Federal de Nutricionistas]. - Brasília, DF. 2015;(3).

9. Recine E, Mortoza AS. Consenso sobre habilidades e competências do nutricionista no âmbito da saúde coletiva. Brasília. Obs Políticas Segur E Nutr. 2014;64.

10. Cervato-Mancuso AM, Tonacio LV, Silva ER da, Vieira VL. A atuação do nutricionista na Atenção Básica à Saúde em um grande centro urbano. Ciênc Saúde Coletiva [Internet]. dezembro de 2012 [citado 13 de agosto de 2020];17(12):3289-300. Disponível em: http://www.scielo.br/scielo.php?script=sci_arttext\&pid=S1413-81232012001200014\&lng=pt\&tlng=pt. https://doi.org/10.1590/ S1413-81232012001200014

11. Mais LA, Domene SMÁ, Barbosa MB, Taddei JA de AC. Formação de hábitos alimentares e promoção da saúde e nutrição: o papel do nutricionista nos Núcleos de Apoio à Saúde da Família - NASF. Rev APS. 2015;abr/jun(18(2)):248-55.

12. Mattos PF, Neves A dos S. A importância da atuação do nutricionista na Atenção Básica à Saúde. Rev Práxis. 2009;(2):11-5.

13. Conselho Federal de Nutricionistas. Nutricionista como agente de promoção da saúde [Internet]. CFN. 2016 [citado 13 de agosto de 2020]. Disponível em: /index.php/nutricao-na-midia/nutricionista-como-agente-de-promocao-da-saude/

14. Conselho Federal de Nutricionistas. RESOLUÇÃO CFN No 600, DE 25 DE FEVEREIRO DE 2018. Texto retificado em 23 de maio de 2018. Dispõe sobre a definição das áreas de atuação do nutricionista e suas atribuições, indica parâmetros numéricos mínimos de referência, por área de atuação, para a efetividade dos serviços prestados à sociedade e dá outras providências. 2018.

15. Brasil. Política Nacional de Alimentação e Nutrição / Ministério da Saúde, Secretaria de Atenção à Saúde. Departamento de Atenção Básica. Série B. Textos Básicos de Saúde. 2013;1. reimpr.(1a):84.

16. Conselho Federal de Nutricionistas. Estatística - Quadro estatistico anual por trimestre. [Internet]. CFN. 2020 [citado 2 de março de 2020]. Disponível em: https://www.cfn.org.br/index.php/estatistica/

17. Brasil. Ministério da Saude. Cadastro Nacional de Estabelecimentos de Saúde [Internet]. 2020 [citado 15 de janeiro de 2020]. Disponível em: http://cnes.datasus.gov.br/pages/profissionais/extracao.jsp

18. IBGE. Tabela 6579: População residente estimada [Internet]. 2020 [citado 4 de março de 2020]. Disponível em: https://sidra.ibge. gov.br/tabela/6579

19. Recine E, Gomes RCF, Fagundes AA, Pinheiro AR de O, Teixeira B de A, Sousa JS de, et al. A formação em saúde pública nos cursos de graduação de nutrição no Brasil. Rev Nutr [Internet]. fevereiro de 2012 [citado 29 de dezembro de 2020];25(1):21-33. Disponível em: http://www.scielo.br/scielo.php?script=sci_arttext\&pid=S1415-52732012000100003\&lng=pt\&tlng=pt. https://doi.org/10.1590/ S1415-52732012000100003.

20. Haddad AE, Morita MC, Pierantoni CR, Brenelli SL, Passarella T, Campos FE. Formação de profissionais de saúde no Brasil: uma análise no período de 1991 a 2008. Rev Saúde Pública [Internet]. junho de 2010 [citado 29 de dezembro de 2020];44(3):383-93. Disponível em: http://www.scielo.br/scielo.php?script=sci_arttext\&pid=S0034-89102010000300001\&lng=pt\&tlng=pt. https://doi. org/10.1590/S0034-89102010005000015.

21. Neves J das, Zanlourensi CB, Domene SMÁ, Batista B, Calado CL de A, Vasconcelos F de AG de. Eighty years of undergraduate education in nutrition in Brazil: An analysis of the 2009-2018 period. Rev Nutr. 2019;32:e180158. http://dx.doi.org/10.1590/1678$9865201932 \mathrm{e} 180158$.

22. Universidade do Estado do Rio de Janeiro. ObservaRH-IMS. Estação de Trabalho da Rede Observatório de Recursos Humanos em Saúde do Instituto de Medicina Social da Universidade do Estado do Rio de Janeiro [Internet]. 2013 [citado 15 de março de 2020]. Disponível em: http://www.obsnetims.org.br/uploaded/4_7_2013_0_Nutricao.pdf

23. Vasconcelos F de AG de, Calado CL de A. Profissão nutricionista: 70 anos de história no Brasil. Rev Nutr. agosto de 2011;24(4):605-17. 24. Gabriel CG, Oliveira JTC de, Silva BL, Fagundes AA, Silva TC, Soar C. Nutritionist's job market: 80 years of history. Rev Nutr. 2019;32:e180162. 
25. Brasil. Lei no 9.394, de 20 de dezembro de 1996. Estabelece as diretrizes e bases da educação nacional. [Internet]. 1996. Disponível em: http://www.planalto.gov.br/ccivil_03/leis/l9394.htm

26. Conselho Federal de Nutricionistas. Perfil dos nutricionistas do Brasil. [Internet]. 2019 [citado 20 de março de 2020]. Disponível em: http://pesquisa.cfn.org.br/.

27. Brasil. Cadastro e-MEC de Instituições e Cursos de Educação Superior. Relatório Cursos de Nutrição. [Internet]. 2020 [citado 20 de março de 2020]. Disponível em: http://emec.mec.gov.br/

28. Pinheiro AR de O, Recine E, Alencar B de, Fagundes AA, Sousa JS de, Monteiro RA, et al. Percepção de professores e estudante em relação ao perfil de formação de nutricionista em saúde pública. Rev Nutr. outubro de 2012;25(5):632-43. http://dx.doi.org/10.1590/ S1415-52732012000500008.

29. Akutsu R de C. Brazilian dieticians: professional and demographic profiles. Rev Nutr. fevereiro de 2008;21(1):7-19. http://dx.doi. org/10.1590/S1415-52732008000100002.

30. Gambardella AMD, Ferreira CF, Frutuoso MFP. Situação profissional de egressos de um curso de nutrição. Rev Nutr. abril de 2000;13(1):37-40. http://dx.doi.org/10.1590/S1415-52732000000100005.

31. Miranda DEG de A, Pereira CHC, Paschoini TB, Quaglio T. O perfil de atuação dos ex-alunos do curso de nutrição de uma universidade do interior paulista. Investigação. 2010;10:54-9. https://doi.org/10.26843/investigacao.v10i2-3.307.

32. Assis AMO, Santos SMC dos, Freitas M do CS de, Santos JM, Silva M da CM da. O Programa Saúde da Familia: contribuições para uma reflexão sobre a inserção do nutricionista na equipe multidisciplinar. Rev Nutr. setembro de 2002;15(3):255-66. http://dx.doi. org/10.1590/S1415-52732002000300001

33. Brasil. Ministério da Saúde. Gabinete do Ministro. PORTARIA No 154, DE 24 DE JANEIRO DE 2008 [Internet]. 2020 [citado 26 de agosto de 2020]. Disponível em: https://bvsms.saude.gov.br/bvs/saudelegis/gm/2008/prt0154_24_01_2008.html

34. Borelli M, Domene SMÁ, Mais LA, Pavan J, Taddei JA de AC, Borelli M, et al. A inserção do nutricionista na Atenção Básica: uma proposta para o matriciamento da atenção nutricional. Ciênc Amp Saúde Coletiva. setembro de 2015;20(9):2765-78. https://doi. org/10.1590/1413-81232015209.13902014.

35. Brasil. Ministério da saúde. Plano nacional de alimentação e nutrição 2015-2020. [Internet]. [citado 14 de abril de 2020]. Disponível em: https://www.minsaude.gov.cv/index.php/documentosite/331-plano-nacional-alimentacao-e-nutricao-2015-2020/file 